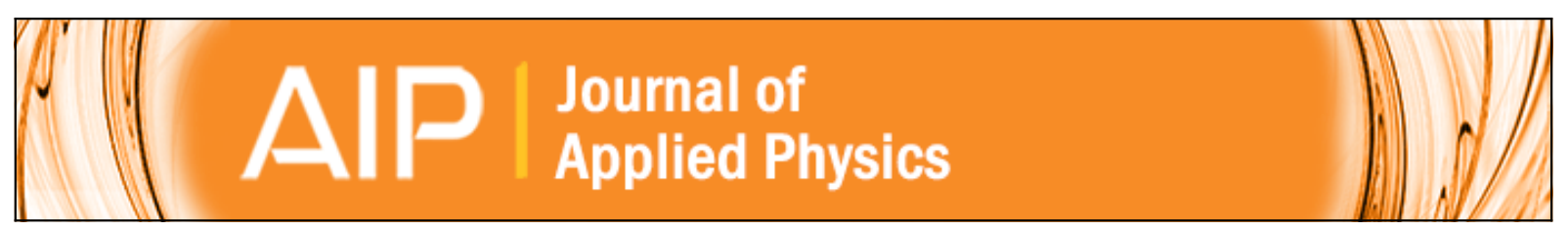

Core level photoemission studies on conducting polypyrrole polymer nanotubes showing switching transitions

I. Sarkar, A. Sarma, M. K. Sanyal, S. Thieß, and W. Drube

Citation: Journal of Applied Physics 114, 163707 (2013); doi: 10.1063/1.4827196

View online: http://dx.doi.org/10.1063/1.4827196

View Table of Contents: http://scitation.aip.org/content/aip/journal/jap/114/16?ver=pdfcov

Published by the AIP Publishing

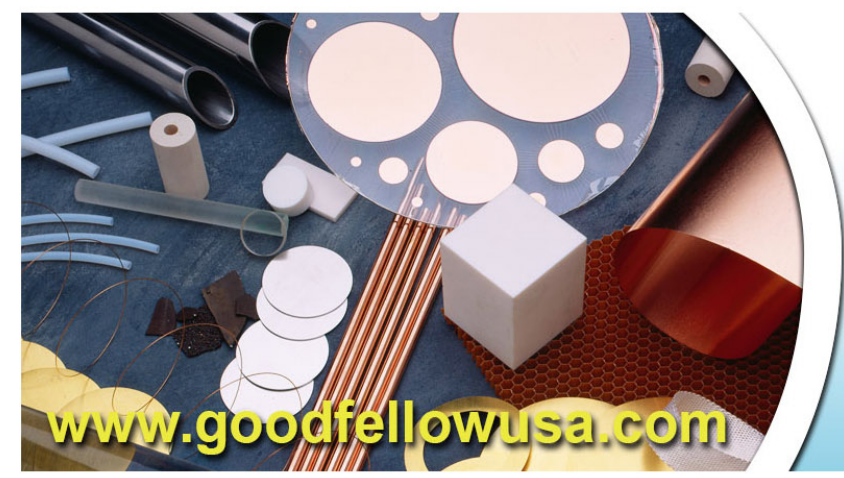

BOIIFEIII

metals • ceramics $\cdot$ polymers composites • compounds • glasses

Save $5 \% \cdot$ Buy online 70,000 products $\cdot$ Fast shipping 


\title{
Core level photoemission studies on conducting polypyrrole polymer nanotubes showing switching transitions
}

\author{
I. Sarkar, ${ }^{1, \text { a) }}$ A. Sarma, ${ }^{2}$ M. K. Sanyal, ${ }^{2}$ S. Thieß, ${ }^{1}$ and W. Drube ${ }^{1}$ \\ ${ }^{1}$ DESY Photon Science, Deutsches Elektronen-Synchrotron, 22603 Hamburg, Germany \\ ${ }^{2}$ Surface Physics Division, Saha Institute of Nuclear Physics, 1/AF Bidhannagar, Kolkata 700064 , India
}

(Received 30 July 2013; accepted 11 October 2013; published online 29 October 2013)

\begin{abstract}
Conducting polypyrrole (PPY) nanotubes is a classical model system for strongly correlated disordered materials showing intriguing switching transitions from low to high conductivity states at low temperature. This switching behaviour can be tuned by incorporating gold nanoclusters to form composite nanotubes (AuPPY). Here, we present core level electronic structure studies on PPY and AuPPY nanotubes with different diameters using hard X-ray photoemission spectroscopy at room temperature. The spectroscopic data provide information on the role of diameter as well as metal cluster incorporation for a modification of the electronic structure of this important class of nanotubes. Furthermore, electrical transport measurements were performed at low temperature to extract the change in localization length and doping level in these nanotubes. Based on these results, we explain the observed electrical transport behavior of these nanotubes as the interplay of disorder and carrier concentration. (c) 2013 AIP Publishing LLC.
\end{abstract}

[http://dx.doi.org/10.1063/1.4827196]

\section{INTRODUCTION}

Conducting polymer is a class of strongly correlated disordered system showing rich transport properties due to the interplay of disorder and carrier concentration. ${ }^{1}$ It is being widely studied for its immense application in organic electronic devices. ${ }^{2}$ It has been found that at reduced dimension these materials show strong improvements in their performance. ${ }^{3,4}$ One particular material in this class that has gained significant importance is polypyrrole (PPY) nanotubes. ${ }^{5} \mathrm{PPY}$ is one of the most widely used active component in various chemical $^{6}$ and biological sensors. ${ }^{7}$ Electrical transport in PPY nanotubes has been a subject of intensive research, ${ }^{3,8,11}$ as it not only provides a model system for testing non-Fermi liquid behaviour in conducting polymers ${ }^{3,12}$ but also holds huge potential in device application, ${ }^{13}$ owing to its ease of preparation, robust environmental stability, and high electrical conductivity. It has been found that PPY nanotubes show a novel resistance switching transition to a high conducting state from a low conducting state. ${ }^{3}$ This transition is observed above a threshold voltage and below a certain transition temperature, which strongly depends upon the diameter of the nanotubes. Such a switching behaviour opens up an enormous scope for device applications. ${ }^{14,15}$ These perspectives motivate further detailed studies to understand the electronic structure of these materials as a function of diameter of the nanotubes.

It has been shown recently that the switching transition can be largely tuned by incorporating gold nanoclusters in the PPY (AuPPY) nanotubes. ${ }^{15}$ This was done by developing a single-step synthesis technique where the polymerization of nanotubes occurs simultaneously with the reduction of gold nanoclusters. This technique yields high quality nanotubes of polypyrrole-gold (AuPPY) nanocomposites, such

\footnotetext{
a)indra7neel@gmail.com
}

that the gold nanoclusters are homogeneously embedded inside the nanotube walls. It was found that depending on the diameter of the nanotubes, the switching transition temperature of the gold incorporated nanotubes increases significantly to above liquid nitrogen temperature, as high as $100 \mathrm{~K}$. This clearly indicates a strong metal-polymer nanotube interaction. It is therefore crucial to study the electronic structure of these composite nanotubes in order to understand their different transport properties as compared to noncomposite nanotubes, which will thus pave way for incorporating this material in real world devices. Although some brief supplementary X-ray photoemission (XPS) data on gold-PPY multi-segmented nanotubes ${ }^{8}$ and on gold nanoparticles reduced by pyrrole ${ }^{9}$ can be found in literature, there exists no electronic structure study using XPS for goldpolypyrrole composite nanotubes.

Here, we present results of a hard X-ray photoemission spectroscopy (HAXPES) study of the electronic structure of PPY nanotubes and gold nanocluster incorporated polypyrrole (AuPPY) nanotubes of varying diameters. HAXPES provides greater depth sensitivity compared to conventional photoemission techniques and allows the investigation of chemical and electronic properties of as-grown bulk materials as well as buried interfaces. The obtained data indicate that PPY nanotubes with smaller diameter show more disorder than larger diameter PPY nanotubes. Furthermore, the incorporation of gold nanoclusters in AuPPY nanotubes is found to lead to less disordered nanotubes, accompanied by a reduction of the carrier concentration. The results demonstrate the role played by confinement effects as well as metal cluster incorporation for engineering the electronic structure of this important class of materials, especially the interplay between disorder and carrier concentration. The obtained spectroscopic data help to explain the modification of the electrical transport properties observed for these nanotubes. Furthermore, we have performed electrical transport measurements on these nanotubes to obtain the 
localization length and density of states at the Fermi level. The transport data are found to be consistent with the obtained spectroscopic results.

\section{EXPERIMENT}

PPY and AuPPY nanotubes were synthesized using a membrane-based synthesis technique to grow polymer nanowires. In this method, polycarbonate membranes having nanopores of varying diameter were used as a template to grow nearly mono-disperse nanotubes. Details of this synthesis have been described elsewhere. ${ }^{15}$ The nanotubes are being formed due to polymerization of pyrrole monomers inside the nanopores of the membrane in the presence of an oxidizing agent. In the case of pure PPY nanotubes, ferric chloride $\left(\mathrm{FeCl}_{3}\right)$ was used as the oxidizing agent. For the synthesis of AuPPY nanotubes, the oxidizing agent consisted of a mixture (1:1 volumetric ratio) of $0.5 \mathrm{M}$ ferric chloride and $3 \mathrm{mM}$ chloroauric acid $\left(\mathrm{HAuCl}_{4}\right)$ solution. The obtained nanotubes were characterized by scanning electron microscopy (SEM), transmission electron microscopy (TEM), and energy dispersive X-ray (EDX) spectroscopy.

HAXPES measurements were carried out at the BW2 X-ray wiggler beamline of the DORIS III storage ring (DESY, Hamburg) choosing a photon energy of $3 \mathrm{keV}$. The total energy resolution estimated is $0.5 \mathrm{eV}$. Small photon energy drifts that may be induced by heat load variations on the double-crystal monochromator were taken care of by monitoring the position of the $\mathrm{Au} 4 \mathrm{f}_{7 / 2}$ line measured from a gold foil on the sample holder. Photoelectrons emitted in the plane of photon polarization at $45^{\circ}$ relative to the polarisation vector were recorded using a SCIENTA SES-200 electron spectrometer. All spectra were fitted using the UNIFIT software. ${ }^{16}$

Low temperature electronic transport measurements were done in Janis $4 \mathrm{~K}$ Closed Cycle Refrigeration Cryostat. Magnetoresistance measurements were performed using 5T Superconducting cryostat magnet of Cryomagnetics, in the presence of magnetic field aligned along the nanotube axis. All the electrical transport measurements were carried out in pseudo four probe and two probe configuration. ${ }^{15}$ Gold contact was sputter deposited on both side of the membrane to connect these nanotubes. It was found that sputter-deposited gold contact gives low contact resistance and small depletion capacitance. ${ }^{10}$ The I-V characteristics was measured by driving current using Keithley 2602 source-meter while measuring voltage using Agilent 34420A nano-voltmeter or Keithley 2001 Multimeter. The Magneto-resistance was measured using Agilent 33220A Function generator, SRS 570 current preamplifier, and SR 830 DSP Lock-in-amplifier. Temperature was controlled by Lakeshore 340 temperature controller with the help of a manganin wire heater $(50 \Omega)$. Before each measurement, the performance of the setup was checked using standard $100 \mathrm{G} \Omega$ resistance. All the instruments were interfaced with a computer via GPIB interface. Labview (National Instruments Corp., Austin, TX) software was used for data acquisition.

\section{RESULTS}

The high quality of the nanotubes was confirmed using SEM (Fig. 1(a)). Furthermore, TEM (inset of Fig. 1(a)) of the inner wall of AuPPY nanotubes showed the presence of highly crystalline nanoclusters embedded in the wall. These clusters consist of $\mathrm{Au}$ atoms as confirmed by the observed lattice spacing and EDX spectroscopy data. The electrical switching transition found in $200 \mathrm{~nm}$ diameter nanotubes of PPY and AuPPY (Fig. 1(b)) exhibits a current increase by orders of magnitude above a certain threshold voltage $\left(\mathrm{V}_{t h}\right)$. When the voltage is reduced, the I-V curve does not follow its original path, rather it traces a hysteresis curve and requires a return voltage $\left(\mathrm{V}_{r e}\right)$ to switch back to the low conducting state. As previously reported, ${ }^{15}$ it was found that the switching voltage is higher for AuPPY than for PPY nanotubes (Fig. 1(b)). The appearance of a switching transition in these materials has been explained using a 1D Wigner crystal model for disordered systems that has characteristics similar to charge density wave (CDW) systems pinned by impurities and defects. ${ }^{3}$ The enhanced conductivity above a threshold electric field (voltage) in the nanotubes is thus explained as being similar to a sliding CDW state where the CDW starts depinning above the threshold voltage, accompanied by a giant increase in conductivity. 3,15

\section{A. HAXPES measurements}

Survey X-ray photoelectron spectra of the PPY nanotubes revealed the presence of carbon and nitrogen as the major constituents along with oxygen and traces of iron and chlorine from the oxidizing agent, additionally gold lines are

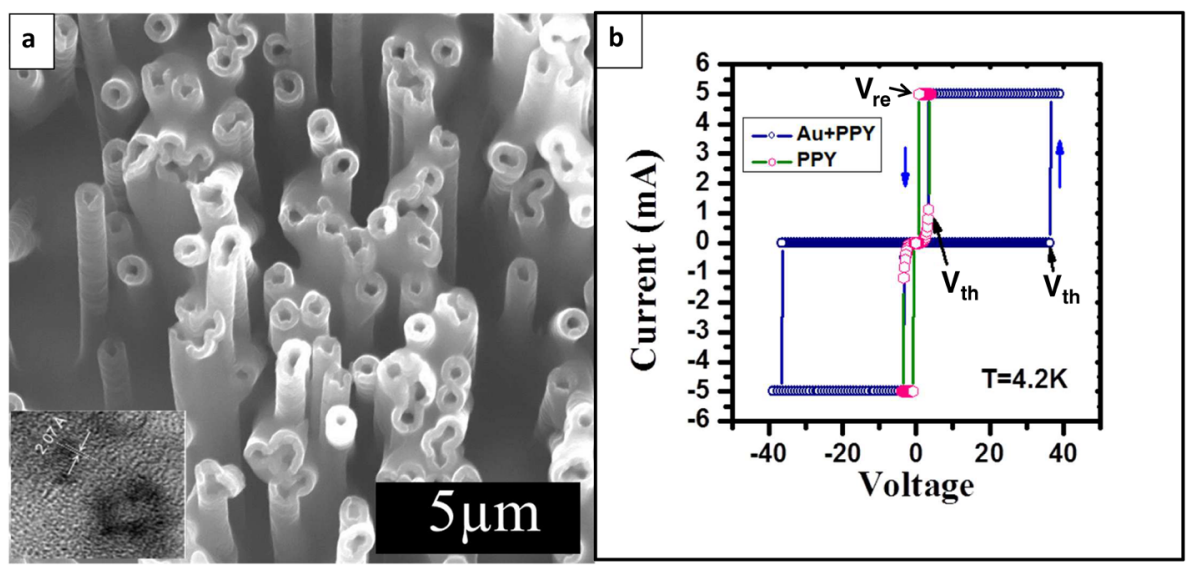

FIG. 1. (a) SEM image of $200 \mathrm{~nm}$ AuPPY nanotubes. The inset shows cross-sectional TEM of the inner wall of an AuPPY nanotube. (b) I-V curve of $200 \mathrm{~nm}$ PPY and AuPPY nanotubes at $4.2 \mathrm{~K}$. 


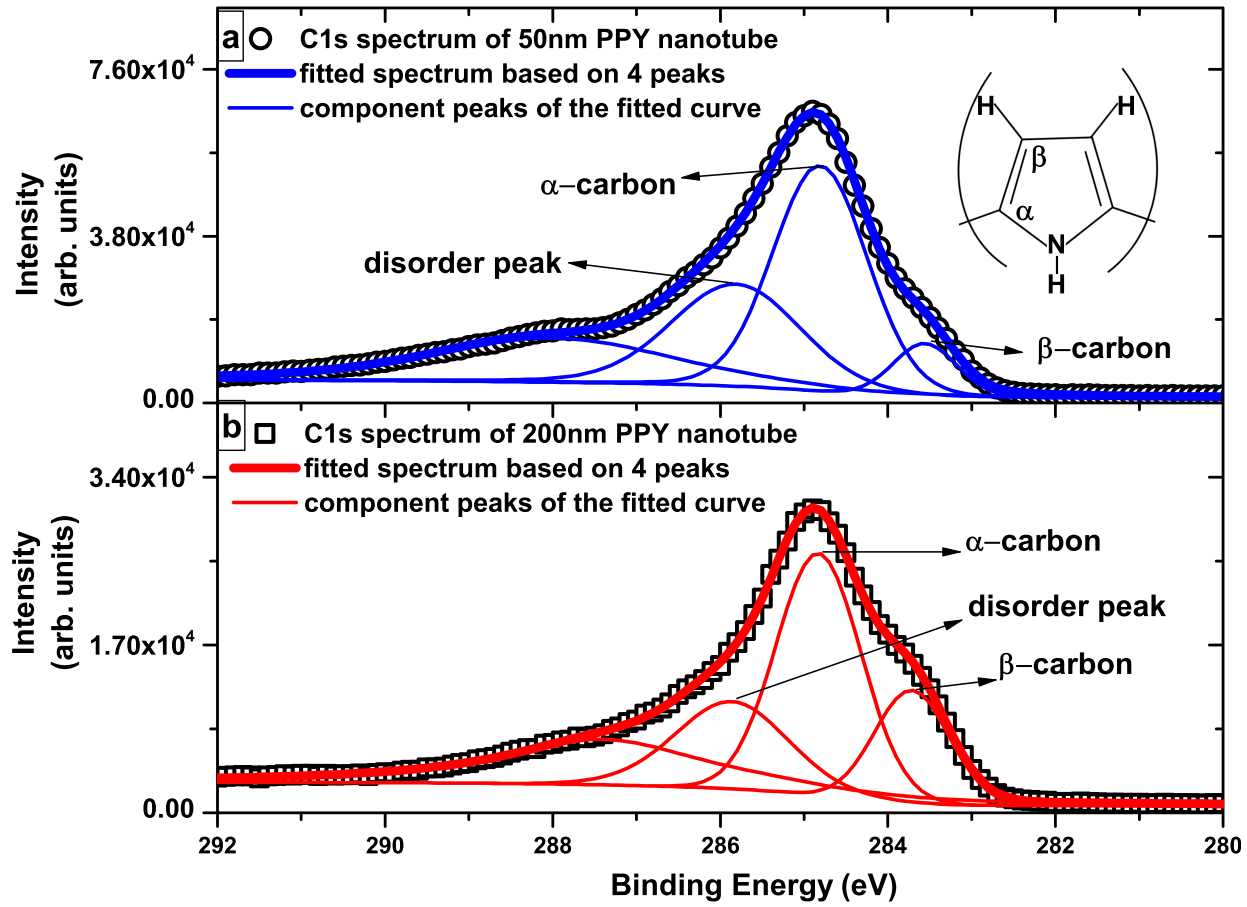

FIG. 2. C1s spectrum of (a) $50 \mathrm{~nm}$ PPY nanotubes, (b) $200 \mathrm{~nm}$ PPY nanotubes. The thick solid line is the fitted curve to the experimental data, assuming four peaks. The thin solid lines correspond to the individual components. The inset in (a) shows structure of pyrrole ring.

clearly observed for AuPPY nanotubes. Figures 2(a) and 2(b) show C1s photoelectron spectra measured for PPY nanotubes with $50 \mathrm{~nm}$ and $200 \mathrm{~nm}$ diameter. Both C 1s spectra are broad and asymmetric and could be fitted by four peaks consistent with previous peak assignments for polypyrrole $^{17-20}$ in bulk and film samples. The lowest binding energy peaks centered around $284.6 \mathrm{eV}$ and $283.6 \mathrm{eV}$ correspond to $\alpha$ and $\beta$ carbon atoms, respectively, in the pyrrole ring ${ }^{17-19}$ as sketched in the inset of Fig. 2(a). In the ideal case of a perfect structure, these two peaks should have the same intensities. ${ }^{18,19}$ However, it is well known that due to disorder and hydrocarbon impurities contributing to the XPS spectrum, the intensity of the $\alpha$ peak is generally enhanced. ${ }^{18,19}$ The third broad peak around $285.8 \mathrm{eV}$ is known to arise due to disorder corresponding to non-ideal $\alpha$ carbon attributed to chain termination, non $\alpha$-bonded carbon atoms, and carbon atoms in partially saturated rings. ${ }^{18,19}$ The peaks at higher binding energy of $288 \mathrm{eV}$ may be attributed to an oxidation of the PPY nanotubes corresponding to the formation of carbonyl $(-\mathrm{C}=\mathrm{O})$, as has been found in oxidized films of PPY. ${ }^{20}$ The comparison of C1s spectra for $50 \mathrm{~nm}$ (Fig. 2(a)) and $200 \mathrm{~nm}$ PPY nanotubes (Fig. 2(b)) shows that for the higher diameter nanotube there is an enhanced density of states around the $\beta$ carbon peak. The peak intensity ratio $\mathrm{C} 1 \mathrm{~s}(\beta) / \mathrm{C} 1 \mathrm{~s}(\alpha)$ is found to increase from 0.25 for $50 \mathrm{~nm}$ PPY nanotube to 0.47 for $200 \mathrm{~nm}$ PPY

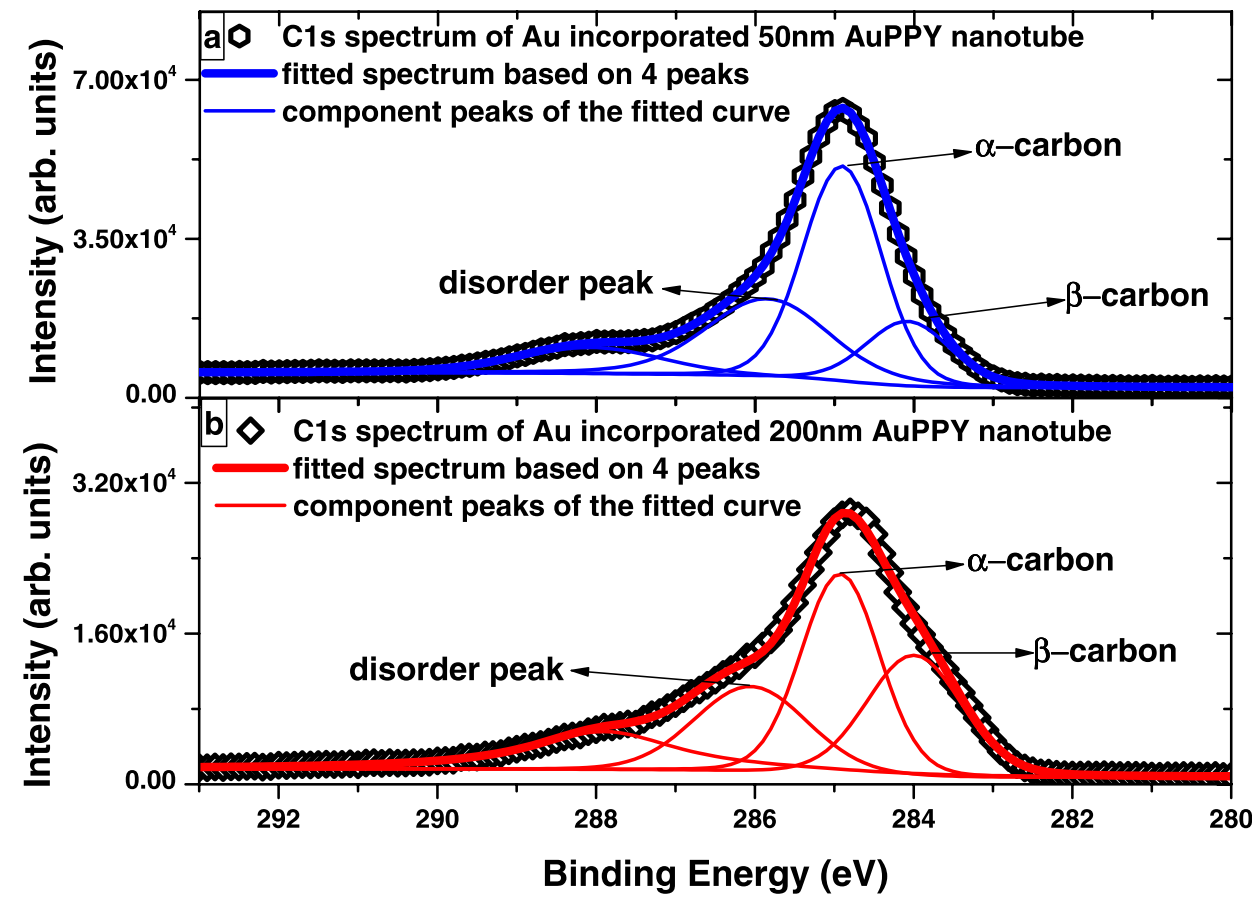

FIG. 3. C1s spectrum of (a) $50 \mathrm{~nm}$ AuPPY nanotubes. (b) $200 \mathrm{~nm}$ AuPPY nanotubes. The thick solid line is the fitted curve to the experimental data, which is the sum curve of four peaks. The thin solid lines are the component peaks of the fitted curve. 


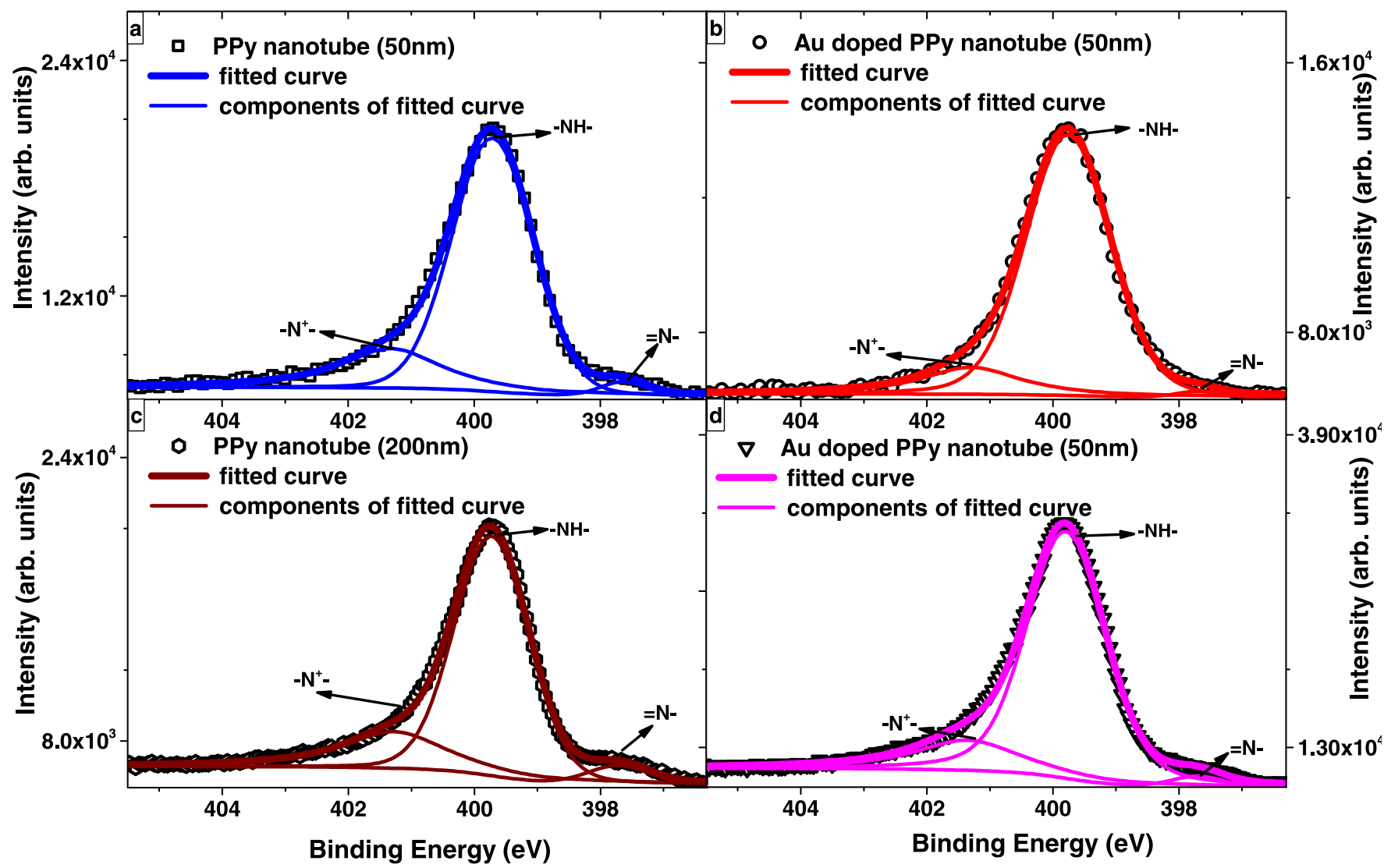

FIG. 4. N1s spectra of (a) $50 \mathrm{~nm}$ PPY nanotubes, (b) $50 \mathrm{~nm}$ AuPPY nanotubes, (c) $200 \mathrm{~nm}$ PPY nanotubes, (d) $200 \mathrm{~nm}$ AuPPY nanotubes, normalized to the peak intensity. The thick solid lines are the fitted curve to the experimental data, which is the sum curve of three peaks. The thin solid lines are the component peaks of the fitted curve.

nanotubes. Also, the area of the disorder related C 1s component around $285.8 \mathrm{eV}$ reduces from $27 \%$ of the total $\mathrm{C} 1 \mathrm{~s}$ spectrum area for $50 \mathrm{~nm}$ nanotubes to $19 \%$ for $200 \mathrm{~nm}$ nanotubes. This strongly indicates less disorder to be present in the larger diameter nanotubes.

C1s spectra for AuPPY nanotubes of $50 \mathrm{~nm}$ and $200 \mathrm{~nm}$ diameter are shown in Fig. 3. It was found that at a fixed diameter, AuPPY nanotubes have higher $\mathrm{C} 1 \mathrm{~s}(\beta) / \mathrm{C} 1 \mathrm{~s}(\alpha)$ peak ratio compared to pure PPY nanotubes. For $50 \mathrm{~nm}$ nanotubes, this ratio increases from 0.25 for PPY to 0.33 for AuPPY, while for $200 \mathrm{~nm}$ it increases from 0.47 to 0.6 . Thus, there is a clear reduction of disorder in AuPPY nanotubes compared to bare PPY nanotubes.

Figure 4 shows nitrogen N1s spectra for PPY and AuPPY nanotubes. All spectra could be fitted with three components corresponding to amine-like $\mathrm{N}(-\mathrm{NH}-)$ atoms, imine-like $\mathrm{N}(=\mathrm{N}-)$ atoms, and positively charged aminelike $\mathrm{N}\left(-\mathrm{N}^{+}\right)$atoms, typical of the polypyrrole spectrum. ${ }^{8,21}$ For PPY nanotubes, a pronounced shoulder is observed at lower binding energy around $397.7 \mathrm{eV}$ corresponding to $(=\mathrm{N}-)$, which is larger compared to that in AuPPY. For $50 \mathrm{~nm}$ diameter nanotubes, the area of the $(=\mathrm{N}-)$ component is found to reduce from $2.4 \%$ of the total $\mathrm{N} 1 \mathrm{~s}$ spectrum of PPY nanotubes to $1 \%$ for AuPPY nanotubes, while for $200 \mathrm{~nm}$ diameter it reduces from $4.3 \%$ for PPY to $2.1 \%$. The reduced density of imine-like $\mathrm{N}$ atoms in AuPPY is due to the protonation of $(=\mathrm{N}-)$ atoms in the presence of chloroauric acid to form (-NH-) atoms, whereby $\mathrm{HAuCl}_{4}$ releases $\mathrm{H}$ atoms that attach to $(=\mathrm{N}-)$ atoms. Such protonation of imine atoms to form amine-like $\mathrm{N}$ atoms in PPY films in the presence of acid solution is well-known. ${ }^{22}$ This leads to a reduction of gold atoms in the process. Similar protonation of nitrogen atoms for an reduction of gold from chloroauric acid has been observed in PPY thin films. ${ }^{23}$

Furthermore, it is found that the area of the $\left(-\mathrm{N}^{+}\right)$-component in the N1s spectrum decreases for AuPPY compared to PPY nanotubes of the same diameter, namely from $19.4 \%$ (PPY) to $12.8 \%$ (AuPPY) for $50 \mathrm{~nm}$ nanotubes

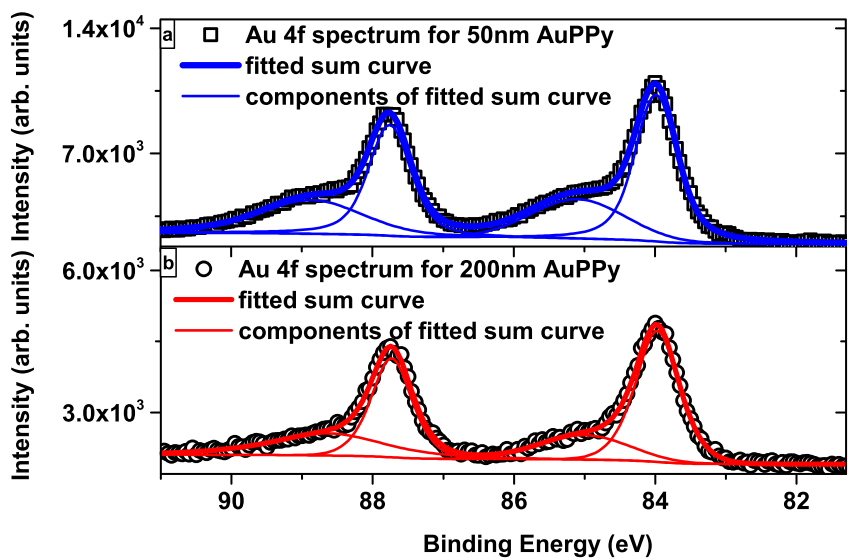

FIG. 5. Au 4f spectra of (a) $50 \mathrm{~nm}$ AuPPY nanotubes. (b) $200 \mathrm{~nm}$ AuPPy nanotubes. The thick solid lines are the fitted curves to the experimental data, corresponding to the sum curve of two components. The thin lines are the component peaks of the fitted curve. 

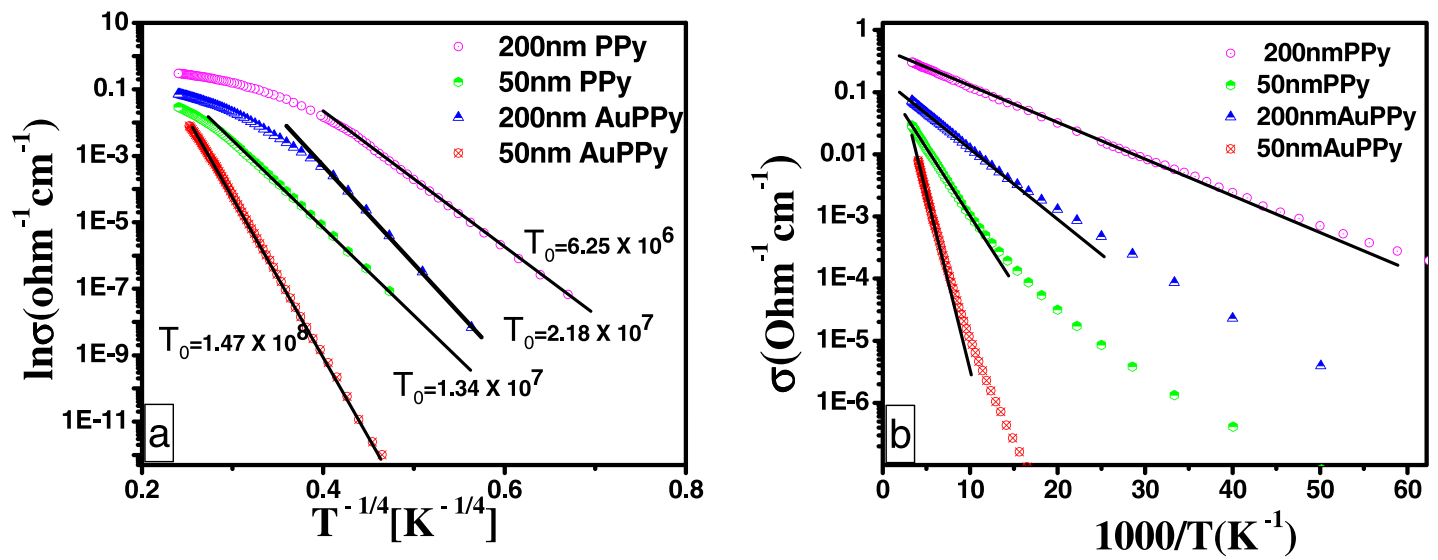

FIG. 6. (a) $\ln (\sigma)$ vs $\mathrm{T}^{-1 / 4}$ plot, showing 3D-VRH behaviour of $200 \mathrm{~nm}$ PPy, $50 \mathrm{~nm}$ PPy, $200 \mathrm{~nm}$ AuPPy, and $50 \mathrm{~nm}$ AuPPy at low temperature. The solid line shows 3D-VRH fitting to the low temperature data using Eq. (1). The value of $\mathrm{T}_{0}$ indicated in each graph corresponds to Mott's temperature. (b) $\sigma$ vs $\mathrm{T}^{-1}$ plot showing thermally activated behaviour (solid line) at higher temperatures.

and from $19.8 \%$ (PPY) to $15.4 \%$ (AuPPY) for $200 \mathrm{~nm}$ diameter material. A decrease of the $\mathrm{N}^{+}$-component has been ascribed to a reduction of the doping level in nanotubes, ${ }^{8}$ and the present data therefore suggest a reduction of the carrier concentration in gold incorporated nanotubes.

For AuPPY nanotubes, the Au 4f core level spectra reveal the well-known doublet structure corresponding to $4 \mathrm{f}_{7 / 2}$ and $4 \mathrm{f}_{5 / 2}$ at $84.0 \mathrm{eV}$ and $87.7 \mathrm{eV}$ binding energy ${ }^{9}$ (Fig. 5). However, each component exhibits a pronounced shoulder at higher binding energies. The spectra were thus fitted with two peaks, one corresponding to neutral $\mathrm{Au}^{0}$ and the other to positively charged state of Au. The data show that gold is mostly reduced to the neutral state but some are only partially reduced to positively charged state from chloroauric acid. Comparing the data obtained for $50 \mathrm{~nm}$ and $200 \mathrm{~nm}$ diameter AuPPY (Fig. 5), it is obvious that the ratio of the charge states depends on the diameter and the amount of $\mathrm{Au}^{+}$is less in the $200 \mathrm{~nm}$ material.

\section{B. Electrical transport}

Figure 6 shows results of temperature dependent conductivity $(\sigma)$ measurements of the PPY and AuPPY nanotubes of $50 \mathrm{~nm}$ and $200 \mathrm{~nm}$ diameter. The conductivity shows a linear behaviour of $\ln (\sigma)$ vs $\mathrm{T}^{-1 / 4}$ (solid line in Fig. 6(a)) at low temperatures, whereas at higher temperatures it is a $T^{-1}$ behaviour (solid line Fig. 6(b)). This may be expected in this system, ${ }^{8}$ where charge transport is governed by Variable Range Hopping (VRH) model ${ }^{24}$ at low temperatures while at higher temperatures thermally activated transport dominates. For the PPY nanotubes, the VRH behaviour is found to prevail up to $28 \mathrm{~K}$ and $95 \mathrm{~K}$ for $200 \mathrm{~nm}$ and $50 \mathrm{~nm}$ nanotubes, respectively, while for corresponding diameter AuPPy nanotubes this exists up to $35 \mathrm{~K}$ and $199 \mathrm{~K}$, respectively. The conductivity for a 3D-VRH system is given by ${ }^{24}$

$$
\sigma=\sigma_{0} \exp \left[-\left(T / T_{0}\right)^{1 / 4}\right]
$$

where $T_{0}$ is the characteristic Mott temperature that depends on the inverse of localization length $(\alpha)$ and density of states at Fermi level $\mathrm{N}\left(E_{F}\right)$. It is expressed as ${ }^{24}$

$$
T_{0}=18.1 \alpha^{3} / k_{B} N\left(E_{F}\right) .
$$

Using 3D-VRH fitting (Eq. (1)) to the low temperature data in Fig. 6(a), we obtain $\mathrm{T}_{0}$ values of $6.25 \times 10^{6} \mathrm{~K}$, $1.34 \times 10^{7} \mathrm{~K}, 2.18 \times 10^{7} \mathrm{~K}$, and $1.47 \times 10^{8} \mathrm{~K}$ for $200 \mathrm{~nm}$ PPY, $50 \mathrm{~nm}$ PPY, $200 \mathrm{~nm}$ AuPPY, and $50 \mathrm{~nm}$ AuPPY nanotubes, respectively. Using these values of $\mathrm{T}_{0}$, we can determine the localization length $\left(\alpha^{-1}\right)$, from the magneto-resistance measurements (Fig. 7) of these nanotubes.

In the 3D-VRH region, in the presence of a weak magnetic field (B), the magneto-resistance is known to exhibit a $\mathrm{B}^{2}$ behaviour given by ${ }^{25}$

$$
\ln [R(B) / R(0)]=t_{1}\left(B / B_{C}\right)^{2}\left[T_{0} / T\right]^{1 / 4},
$$

where $\mathrm{t}_{1} \approx 0.1, \mathrm{R}(\mathrm{B})$ and $\mathrm{R}(0)$ denote the resistance in the presence and absence of magnetic field $\mathrm{B}$, respectively. $\mathrm{B}_{C}$ is the critical magnetic field above which a weak magnetic field behaviour given by Eq. (3) does not hold and a $\mathrm{B}^{1 / 3}$ behaviour is observed. It is given by

$$
B c=6 \hbar /\left[e \alpha^{-2}(T 0 / T)^{1 / 4}\right] .
$$

The $\ln (\mathrm{R}(\mathrm{B}) / \mathrm{R}(0)) \quad$ vs $\quad \mathrm{B}^{2}$ plot (Fig. $\left.7(\mathrm{a})\right)$ and $\ln (\mathrm{R}(\mathrm{B}) / \mathrm{R}(0))$ vs $\mathrm{B}^{1 / 3}$ (Fig. $\left.7(\mathrm{~b})\right)$ taken at $25 \mathrm{~K}$ in the 3D-VRH region of PPY and AuPPY nanotubes clearly show $\mathrm{B}^{2}$ and $\mathrm{B}^{1 / 3}$ dependence of magneto-resistance at low and high magnetic field, respectively. Combining these two plots and using Eq. (3) for fitting the low field data in Fig. 7(a), we obtain $\mathrm{B}_{C}$ values of $0.9 \mathrm{~T}, 1.0 \mathrm{~T}, 0.5 \mathrm{~T}$, and $0.4 \mathrm{~T}$ for $200 \mathrm{~nm}$ PPY, $50 \mathrm{~nm}$ PPY, $200 \mathrm{~nm}$ AuPPY, and $50 \mathrm{~nm}$ AuPPY nanotubes, respectively. Using these values of $\mathrm{B}_{C}$ and corresponding values of $\mathrm{T}_{0}$ in Eq. (4), we obtain localization lengths $\left(\alpha^{-1}\right)$ of $14 \mathrm{~nm}( \pm 0.28 \mathrm{~nm}), 12.08 \mathrm{~nm}( \pm 0.24 \mathrm{~nm})$, $16 \mathrm{~nm}( \pm 0.32 \mathrm{~nm})$, and $14.16 \mathrm{~nm}( \pm 0.28 \mathrm{~nm})$ for $200 \mathrm{~nm}$ PPY, $50 \mathrm{~nm}$ PPY, $200 \mathrm{~nm}$ AuPPY, and $50 \mathrm{~nm}$ AuPPy nanotubes, respectively. This clearly indicates that the localization length decreases as the diameter of nanotubes decreases, while it increases on incorporation of gold for a given diameter of the nanotube. The localization length is directly related to the amount of disorder in the nanotubes ${ }^{8}$ and decreases 

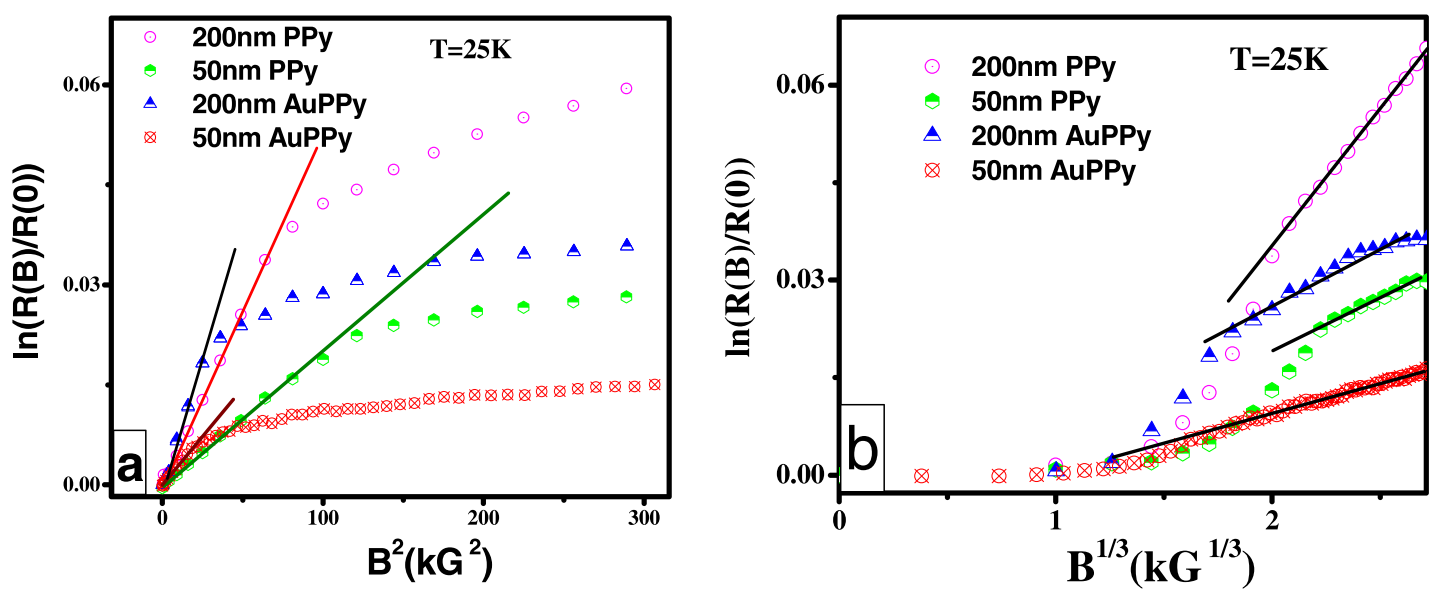

FIG. 7. (a) $\ln [R(B) / R(0)]$ vs $B^{2}$ plot showing, $B^{2}$ dependence (solid line: fit to Eq. (3)) of magneto-resistance at low magnetic field. All the data are taken at a temperature of $25 \mathrm{~K}$ which is within the $3 \mathrm{D}-\mathrm{VRH}$ region of charge transport in the nanotubes. (b) $\ln [\mathrm{R}(\mathrm{B}) / \mathrm{R}(0)]$ vs $\mathrm{B}^{1 / 3}$ plot showing $\mathrm{B}^{1 / 3}$ behaviour (solid line) of magneto-resistance at high magnetic field.

with the increase of disorder. Thus, the disorder increases as the diameter of nanotubes decreases, while it decreases on incorporation of gold for a given nanotube diameter, which is also obtained from the $\mathrm{C} 1 \mathrm{~s}$ photoemission data.

Using the values of $\alpha$ and the corresponding $\mathrm{T}_{0}$ in Eq. (2), we obtain $\mathrm{N}\left(\mathrm{E}_{F}\right)$ values of $1.21 \times 10^{16} \mathrm{eV}^{-1} \mathrm{~cm}^{-3}$, $8.834 \times 10^{15} \mathrm{eV}^{-1} \mathrm{~cm}^{-3}, 2.3 \times 10^{15} \mathrm{eV}^{-1} \mathrm{~cm}^{-3}$, and 4.999 $\times 10^{14} \mathrm{eV}^{-1} \mathrm{~cm}^{-3}$ for $200 \mathrm{~nm}$ PPY, $50 \mathrm{~nm}$ PPY, $200 \mathrm{~nm}$ AuPPY, and $50 \mathrm{~nm}$ AuPPY nanotubes, respectively. In the case of PPY films and nanotubes, it is known that a change in the doping level leads to the change in the density of states at the Fermi level $\mathrm{N}\left(\mathrm{E}_{F}\right) .{ }^{8,26}$ Thus, our results show a reduction in doping level on reduction of the diameter of PPY nanotubes as well as on gold incorporation. These findings are in concomitance to the findings of doping level change obtained from $\mathrm{N}$ 1s photoemission measurements.

\section{DISCUSSION}

The results obtained from this photoemission study combined with electrical transport measurement give crucial information regarding disorder, carrier concentration, and multiple charge states of $\mathrm{Au}$ in the nanotubes and provide a possible explanation of the observed modifications in the switching transition temperature of AuPPY nanotubes. ${ }^{15}$ Furthermore, the results may also explain the change in switching voltage observed in AuPPY compared to PPY nanotubes. ${ }^{15}$ It is known that an increase (decrease) of the switching voltage requires either an increase (decrease) of the pinning strength or an increase (decrease) of the number of pinning sites. ${ }^{3}$ Thus, a modification of the switching voltage cannot be explained by a single parameter.

In the nanotubes, the pinning sites are disorder related defects. The reduction of disorder in AuPPY nanotubes as concluded from the $\mathrm{C} 1 \mathrm{~s}$ photoemission spectra and electrical transport data may explain the recent electrical transport results that show an increase in switching transition temperature in AuPPY nanotubes compared to PPY nanotubes of the same diameter. ${ }^{15}$ This is because conducting polymer nanotubes are chains of metal fibers or strands interrupted by dis$\operatorname{order}^{11,27}$ that show an electron ordering when the long range electronic correlation percolates across these strands over the nanotubes. ${ }^{3,28}$ As AuPPY nanotubes have reduced disorder, there is a higher possibility of having longer uninterrupted strands of ordered nanotubes over which long range electronic correlations can be established at higher temperatures. On the other hand, in case of pure PPY nanotubeshaving more disorder-comparatively smaller uninterrupted strands can be established. Therefore, PPY nanotubes would require lower temperatures for establishing long range electronic correlation compared AuPPY, as is expected for a system with smaller uninterrupted strands. ${ }^{28}$

On the other hand, the analysis of N1s spectra and transport measurements show that AuPPY nanotubes exhibit reduced carrier concentration compared to PPY nanotubes of same diameter. It is therefore possible that the effect of reduced disorder observed in AuPPY nanotubes is overcompensated by a reduction of carrier concentration, thereby reducing the overall conductivity in AuPPY. This would be consistent with the current vs voltage data for AuPPY and PPY nanotubes shown in Fig. 1(b), where at a given voltage PPY shows higher current than AuPPY. A reduced carrier concentration would also lead to weaker screening of pinning sites enhancing the pinning strength ${ }^{29}$ possibly overcompensating the effect of reduced disorder which otherwise indicates a lower number of pinning sites. This in turn should result in higher switching voltages compatible with the observation that the switching voltage (Fig. 1(b)) is higher for AuPPY than PPY nanotubes of same diameter. This has also been observed in earlier electrical transport measurements. ${ }^{15}$

\section{CONCLUSION}

Using hard X-ray photoemission, the core level electronic structure of conducting polymer nanotubes of polypyrrole and $\mathrm{Au}$ nanocluster incorporated nanocomposite polypyrrole was studied for different diameters. In addition, transport measurements were done to compliment the spectroscopic results. The results show that the introduction of Au nanoclusters in the nanotubes as well as larger diameters of the nanotubes lead to less disordered conducting polymer 
chains, which is a key factor for their electrical transport behaviour. Furthermore, the carrier concentration is found to be reduced in AuPPY compared to PPY nanotubes for a given diameter. Based on these results, the enhancement of the switching transition temperature of AuPPY compared to PPY nanotubes is explained by a reduced number of pinning sites going along with a reduction of disorder. On the other hand, the lower carrier concentration deduced from the N1s spectra of AuPPY nanotubes leads to a reduced screening of pinning sites causing enhanced pinning strength, which in turn would explain the increase of the switching voltage in AuPPY. The present spectroscopic results along with the transport measurements add to a deeper understanding of how disorder and carrier concentration may affect the electrical transport properties of these nanotube materials. The type of study presented here is a powerful diagnostic tool for engineering the behaviour of conducting polymer nanotubes. It provides valuable information for a better understanding of the role of dimensionality and metal incorporation for tuning the interplay of carrier concentration and disorder.

\section{ACKNOWLEDGMENTS}

Financial support received from the Indian Department of Science \& Technology (DST) provided within the framework of the India-Germany Collaboration Project India@DESY is acknowledged.

${ }^{1}$ K. Lee, S. Cho, S. H. Park, A. J. Heeger, C.-W. Lee, and S.-H. Lee, Nature 441, 65 (2006).

${ }^{2}$ A. J. Heeger, Rev. Mod. Phys. 73, 681 (2001).

${ }^{3}$ A. Rahman and M. K. Sanyal, Phys. Rev. B 76, 045110 (2007).

${ }^{4}$ Y. Z. Longa, M. M. Lia, C. Gub, M. Wanc, J. L. Duvaild, Z. Liue, and Z. Fanf, Prog. Polym. Sci. 36, 1415 (2011).
${ }^{5}$ T. V. Vernitskaya and O. N. Efimov, Russ. Chem. Rev. 66, 443 (1997).

${ }^{6}$ J. Janata and M. Josowicz, Nature Mater. 2, 19 (2003).

${ }^{7}$ S. K. Mishra, R. Pasricha, A. M. Biradar, and Rajesh, Appl. Phys. Lett. 100, 053701 (2012).

${ }^{8}$ L. Gence, S. Faniel, C. Gustin, S. Melinte, V. Bayot, V. Callegari, O. Reynes, and S. Demoustier-Champagne, Phys. Rev. B 76, 115415 (2007).

${ }^{9}$ M. C. Henry, C. C. Hsueh, B. P. Timko, and M. S. Freund, J. Electrochem. Soc. 148, D155 (2001).

${ }^{10}$ A. Rahman and M. K. Sanyal, Appl. Phys. Lett. 94, 242102 (2009).

${ }^{11}$ D. Talukdar, U. N. Nandi, A. Poddar, P. Mandal, and K. K. Bardhan, Phys. Rev. B 86, 165104 (2012).

${ }^{12}$ V. Gomis, N. Ferrer-Anglada, B. Movaghar, J. M. Ribo, Z. El-Hachemi, and S. H. Jhang, Phys. Rev. B 68, 115208 (2003).

${ }^{13}$ Y. Berdichevsky and Y.-H. Lo, Adv. Mater. 18, 122 (2006).

${ }^{14}$ A. Rahman and M. K. Sanyal, Adv. Mater. 19, 3956 (2007).

${ }^{15}$ A. Sarma, M. K. Sanyal, A. Rahman, and B. Satpati, J. Appl. Phys. 112, 044304 (2012).

${ }^{16}$ See http://unifit-software.de for detailes of the UNIFIT software.

${ }^{17}$ L. Atanasoska, K. Naoi, and W. H. Smyrl, Chem. Mater. 4, 988 (1992).

${ }^{18}$ P. Pfluger and G. B. Street, J. Chem. Phys. 80, 544 (1984).

${ }^{19}$ G. B. Street, T. C. Clarke, R. H. Geiss, V. Y. Lee, A. Nazzal, P. Pfluger, and J. C. Scott, J. Phys. Colloq. 44, C3-599 (1983).

${ }^{20}$ A. Jaramillo, L. D. Spurlock, V. Young, and A. B. Toth, Analyst 124, 1215 (1999)

${ }^{21}$ E. Pigois-Landureau, Y. F. Nicolau, and M. Delamar, Synth. Met. 72, 111 (1995).

${ }^{22}$ O. Inganäs, R. Erlandsson, C. Nylander, and I. Lundström, J. Phys. Chem. Solids 45, 427 (1984)

${ }^{23}$ K. G. Neoh, T. T. Young, N. T. Looi, and E. T. Kang, Chem. Mater. 9, 2906 (1997).

${ }^{24}$ T. G. Castner, in Hopping Transport in Solids, edited by M. Pollak and B. I. Shklovskii (Elsevier, Holland, 1990).

${ }^{25}$ B. I. Shklovskii and A. L. Efros, Electronic Properties of Doped Semiconductors (Springer, Berlin, 1984).

${ }^{26}$ I. N. Hulea, H. B. Brom, A. K. Mukherjee, and R. Menon, Phys. Rev. B 72, 054208 (2005).

${ }^{27}$ Q. Li, L. Cruz, and P. Phillips, Phys. Rev. B 47, 1840 (1993).

${ }^{28}$ M. M. Fogler, S. Teber, and B. I. Shklovskii, Phys. Rev. B 69, 035413 (2004).

${ }^{29}$ D. Starešinic, S. V. Zaitsev-Zotov, N. I. Baklanov, and K. Biljaković, J. Chem. Phys. 128, 094501 (2008). 